\title{
CRESCIMENTO E EFICIÊNCIA FOTOSSINTÉTICA DE USO DO NITROGÊNIO E FÓSFORO EM ESPÉCIES FLORESTAIS DA AMAZÔNIA NA FASE JUVENIL ${ }^{1}$
}

\author{
Keila Rêgo Mendes², Ricardo A.Marenco ${ }^{3}$ e Nilvanda dos Santos Magalhães ${ }^{4}$
}

\begin{abstract}
RESUMO - Na Floresta Amazônica, a fotossíntese e o crescimento das árvores podem ser limitados pela disponibilidade de nutrientes. O objetivo deste trabalho foi determinar o efeito do conteúdo de nitrogênio $(\mathrm{N})$ e fósforo $(\mathrm{P})$ foliar na fotossíntese e a eficiência do uso destes nutrientes nas taxas fotossintéticas, bem como avaliar o efeito da luminosidade no crescimento em 10 espécies florestais da Amazônia Central. Os dados foram coletados em janeiro de 2008. A capacidade fotossintética $\left(A_{\mathrm{pot}}\right)$ foi positivamente relacionada com o conteúdo de $\mathrm{N}$ e $\mathrm{P}$, mas a capacidade fotossintética da folha foi responsiva apenas quanto à eficiência no uso do P. Os conteúdos de $\mathrm{N}$ e $\mathrm{P}$ aumentaram com a irradiância do sub-bosque. Observou-se relação positiva entre o conteúdo de $\mathrm{N}$ e a relação N/P, mas a razão N/P não teve efeito na eficiência do uso do P. A área foliar específica Correlacionou negativamente com $\mathrm{N}$ e $\mathrm{P}$, e a eficiência no uso do $\mathrm{N}$ diminuiu com o aumento da razão N/P, que não teve efeito na eficiência do uso do P. O crescimento em diâmetro aumentou com a luminosidade do sub-bosque. Conclui-se que as arvoretas têm alta sensibilidade às variações no ambiente luminoso e o $\mathrm{P}$ é utilizado de forma altamente eficiente nas espécies estudadas.
\end{abstract}

Palavras-chave: Área foliar específica; Irradiância do sub-bosque; Teor de nutrientes foliar

\section{GROWTH AND PHOTOSYNTHETIC USE EFFICIENCY OF NITROGEN AND PHOSPHORUS IN SAPLINGS OF AMAZONIAN TREE SPECIES}

\begin{abstract}
In the Amazon Rainforest, photosynthesis and tree growth may be limited by the availability of nutrient. Thus, the objectives of this study were to determine the effect of leaf $N$ and $P$ content on photosynthesis, and to assess plant growth in response to understory light in ten tree species in Central Amazonia. Data were collected in January 2008. The photosynthetic capacity $\left(A_{p o}\right)$ of leaves positively increased with foliar $N$ and $P$ content, but it was only responsive to $P$ use efficiency. The contents of $N$ and $P$ increased as understory irradiance increased. In addition, we found a positive relationship between $N$ content and the N/P ratio. On the contrary, the relationship between $P$ content and the N/P ratio was negative. Specific leaf area was negatively correlated with $N$ and $P$. $N$ use efficiency declined with increases with the N/P ratio, but the $N / P$ ratio had no significant effect on $P$ use efficiency. Growth in diameter increased with understory light. In conclusion, saplings were highly sensitive to variations in light intensity, and P was used with high efficiency in studied species.
\end{abstract}

Keywords: Specific leaf area; Understory irradiance; Leaf nutrient content.

\section{INTRODUÇÃO}

Há crescente preocupação sobre como mudanças globais derivadas do aumento da concentração de $\mathrm{CO}_{2}$ na atmosfera podem influenciar o clima da Região Amazônica. Alguns modelos climáticos prognosticam aumentos no período de seca (COX et al., 2004). Num estudo prévio, Mendes e Marenco (2010) observaram que a capacidade fotossintética aparentemente não é influenciada pela sazonalidade das chuvas na Amazônia Central. Contudo, ainda não se sabe como as variações nos teores de elementos minerais, como nitrogênio

\footnotetext{
${ }^{1}$ Recebido em 12.04.2011 aceito para publicação em 25.06.2013.

${ }^{2}$ Programa de Pós-graduação em Botanica no Instituto Nacional de Pesquisas da Amazônia, INPA, AM, Brasil. E-mail: $<$ keila.inpa@gmail.com>.

${ }^{3}$ Coordenação em Dinâmica Ambiental, INPA, Manaus, AM, Brasil. E-mail: <rmarenco@inpa.gov.br>.

${ }^{4}$ Programa de Pós-graduação em Ciências de Florestas Tropicais, INPA. E-mail: nilvandamagalhaes@gmail.com;
}

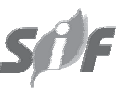

Revista Árvore, Viçosa-MG, v.37, n.4, p.707-716, 2013 
$(\mathrm{N})$ e fósforo $(\mathrm{P})$, podem influenciar a capacidade fotossintética de árvores de dossel na fase juvenil.

Diversos estudos apontam que geralmente existe forte relação entre a fotossíntese e a concentração de $\mathrm{N}$ foliar, pois esse nutriente é componente essencial da enzima ribulose 1,5 bifosfato carboxilase/oxigenase (Rubisco), enzima-chave na fixação de carbono (KITAJIMA et al., 1997; FYLLAS et al., 2009). A importância do $\mathrm{P}$ está relacionada à sua participação na síntese de proteínas e no balanço energético da planta, existindo relação positiva entre taxa de fotossíntese e teor de P foliar (RAAIMAKERS et al., 1995). Além da disponibilidade de nutrientes, a proporção adequada entre os nutrientes essenciais também é importante fator que pode interferir no ganho de biomassa e nas características fotossintéticas (GÜSEWELL, 2004; NEVES et al., 2004; BARROSO et al., 2005). As relações entre as concentrações de N, P e razão N/P dependem da disponibilidade de nutrientes e das condições durante o crescimento, acreditando-se que a relação N/P acima de 16 seja indicativo de restrição na disponibilidade deP(KOERSELMAN; MEULEMAN, 1996; GÜSEWELL, 2004 ).

Vários estudos em florestas tropicais têm relatado diversos efeitos da luminosidade nos processos fisiológicos (DENSLOW et al., 1990; MENDES; MARENCO, 2010; NASCIMENTO; MARENCO, 2013). Entretanto, não se sabe como mudanças da luminosidade no sub-bosque da floresta podem influenciar nas concentrações de $\mathrm{N}$ e $\mathrm{P}$ foliar e na eficiência do uso do $\mathrm{N}$ e P foliar na Amazônia Central. Neste estudo, partiu-se da premissa de que a fotossíntese pode ser limitada pelos conteúdos de $\mathrm{N}$ e $\mathrm{P}$ foliar, assim como a eficiência no uso do $\mathrm{N}$ e P. Assim, os objetivos deste trabalho foram determinar o efeito do conteúdo de $\mathrm{N}$ e P foliar na fotossíntese e a eficiência do uso do $\mathrm{N}$ e $\mathrm{P}$ nas taxas fotossintéticas, bem como avaliar o efeito da luminosidade no crescimento em espécies florestais da Amazônia.

\section{MATERIAL E MÉTODOS}

\section{1. Área de estudo e material vegetal}

O estudo foi realizado na Estação Experimental de Silvicultura Tropical (Núcleo ZF2) do Instituto Nacional de Pesquisas da Amazônia (INPA), localizado a $60 \mathrm{~km}$ ao Norte de Manaus (02 36' $21^{\circ}$ " S; $60^{\circ} 08^{\prime}$ $\left.11^{\mathrm{N}} \mathrm{W}\right)$, em área de Floresta Nativa de Terra Firme.
O clima da região é equatorial úmido, tipo "Am”, na classificação de Köppen (KÖPPEN, 1936), sendo a precipitação anual de 2.240 mm (DIAS, 2009). No estudo, utilizaram-se 10 espécies nativas da Amazônia Central, Chimarrhis duckeana del Prete (Rubiaceae), Ecclinusa guianensis Eyma (Sapotaceae), Erythroxylum amplum Bth. (Erythroxylaceae), Licania oblongifolia Standl. (Chrysobalanaceae), Pouteria guianensis Aubl. (Sapotaceae), Rinorea racemosa (Mart. et Zucc.) O. Ktz. (Violaceae), Sorocea guilleminiana Gaudich. (Moraceae), Tetragastris unifoliolata (Engl.) Cuatr. (Burseraceae), Virola calophylla (Spruce) Warb. (Myristicaceae), Zygia juruana (Harms) L. Rico (Fabaceae), com três repetições cada, uma planta por repetição (devido à impossibilidade de encontrar maior número de plantas da mesma espécie na área de estudo), totalizando 30 arvoretas de 1 a 3 m de altura. A importância das espécies estudadas foi descrita previamente por Mendes e Marenco (2010).

\subsection{Nutrientes (N e P), fotossíntese e área foliar específica}

A eficiência no uso de nutrientes ( $\mathrm{N}$ e P) foi determinada por meio da relação entre quantidade de carbono fixado via fotossíntese (capacidade fotossintética, $A_{\text {pot }}$ ) e o conteúdo de nutrientes foliar (N e P). $A_{\text {pot }}$ foi definida como a taxa de fotossíntese em condições de $\mathrm{CO}_{2}$ e luz saturante (LAWLOR; CORNIC, 2002).

As taxas fotossintéticas $\left(A_{\text {pot }}\right)$ foram mensuradas utilizando um analisador de gás infravermelho (Li-6400, Li-Cor, EUA) em duas folhas por planta e três plantas por espécie. $A_{\text {pot }}$ foi determinada em $\left[\mathrm{CO}_{2}\right]$ saturado de $2.000 \mu \mathrm{mol} \mathrm{mol}^{-1}$, luz saturante $\left(1.000 \mu \mathrm{mol} \mathrm{m}{ }^{-2} \mathrm{~s}^{-1}\right)$ e temperatura ambiente $\left(28 \pm 2{ }^{\circ} \mathrm{C}\right)$. Os dados de fotossíntese foram coletados em janeiro de $2008 \mathrm{em}$ folhas maduras, totalmente expandidas e com bom aspecto fitossanitário.

Para determinação dos conteúdos de nitrogênio (N) e fósforo (P) foliar, utilizaram-se duas a oito folhas por planta em função do tamanho, coletadas em janeiro de 2008 após a medição da fotossíntese. As folhas foram desidratadas em estufa a $72^{\circ} \mathrm{C}$ até massa constante e moídas, sendo quantificados os teores de nitrogênio (N) e fósforo (P). O conteúdo de nitrogênio foliar foi determinado pelo método Kjeldahl. O conteúdo de fósforo foliar foi determinado por calorimetria e as leituras das absorbâncias, feitas a $660 \mathrm{~nm}$, usando-se molibdato de amônio e ácido ascórbico 3\% (SILVA, 2009). Aárea 
foliar específica (AFE) foi determinada como a razão entre a área e a massa foliar. A área foliar foi determinada utilizando um integrador de área (Li-3000A, Li-Cor, EUA), enquanto a massa foliar, com o uso de uma balança de precisão. Os valores SPAD foram medidos com um clorofilômetro (SPAD-502, Minolta, Japão) em cinco pontos, em cada lado da nervura central da folha, na face adaxial. AAFE e os valores SPAD foram determinados nas mesmas folhas utilizadas para análises de nutrientes (nitrogênio e fósforo).

\subsection{Condições ambientais}

A fração de céu visível (FCV) abaixo do dossel da floresta foi determinada usando um analisador do dossel (LAI-2000, Li-Cor, EUA) e calculada como o somatório das aberturas e miniaberturas no dossel da floresta, conforme previamente descrito (MENDES; MARENCO, 2010). A radiação fotossinteticamente ativa (RFA) acima do dossel da floresta $\left(\right.$ RFA $_{\text {dos }}$ ) foi mensurada numa torre de observação a $40 \mathrm{~m}$ de altura, utilizando um sensor quântico (Li-190 SA, Li-Cor, NE, EUA) acoplado a uma Datalogger programado para armazenar dados a cada $15 \mathrm{~min}$. A irradiância diária do sub-bosque $\left(\mathrm{RFA}_{\text {sub }}\right.$ ) foi estimada como o produto da FCV obtido para cada planta (média de seis leituras da FCV por planta) e a média de RFA $_{\text {dos }}$ do mês de janeiro de 2008 $\left(\mathrm{RFA}_{\mathrm{sub}}=\mathrm{FCV} \times \mathrm{RFA}_{\mathrm{dos}}\right)$. Para validar o método, um sensor quântico (Li-190 SA, Li-Cor) foi também instalado no sub-bosque, numa planta escolhida ao acaso, para coleta de dados nos mesmos intervalos daquele localizado na torre de observação. Dados de umidade e temperatura no sub-bosque também foram coletados em intervalos de $30 \mathrm{~min}$, utilizando um sensor de umidade e temperatura (Humitter 50Y, Vaisala, Oyj, Finlândia) acoplado a um Datalogger (Li-1400, Li-Cor).

\subsection{Crescimento das arvoretas}

O incremento anual em diâmetro (IAD) foi medido a $50 \mathrm{~cm}$ acima do coleto, tanto no sentido norte-sul quanto no leste-oeste (para obtenção da média do diâmetro), utilizando-se um paquímetro digital. Para medir o incremento anual em altura (IAA), foi utilizada uma trena. Os dados de crescimento foram coletados mensalmente, no período de janeiro de 2007 a dezembro de 2008, totalizando 24 meses. Ao final do período de estudo, foi determinado o incremento anual em altura (IAA) e diâmetro (IAD).

\subsection{Análise estatística}

Utilizou-se o delineamento experimental inteiramente casualizado. As diferenças entre espécies nas variáveis estudadas foram examinadas mediante a análise de variância (ANOVA), utilizando o teste de Tukey $(\mathrm{P} \leq 0,05)$ para a separação de médias. As variáveis quantitativas ( $\mathrm{N}$ e $\mathrm{P}$ foliar, eficiência no uso do $\mathrm{N}$ e $\mathrm{P}$, área foliar específica) foram submetidas à análise de regressão, para examinar o efeito da irradiância estimada do subbosque nas variáveis quantitativas.

\section{RESULTADOS}

\subsection{Condições ambientais e crescimento das arvoretas}

Os valores de irradiância diária no sub-bosque $\left(\mathrm{RFA}_{\text {sub }}\right.$ ) estimados como o produto de FCV e RFA foram similares aos registrados pelo sensor quântico (Figura 1A), mostrando a validade do método. Acima do dossel da floresta, a irradiância média no período de estudo foi de $20,83 \mathrm{~mol} \mathrm{~m}^{-2} \mathrm{dia}^{-1}$, enquanto no subbosque a irradiância estimada $\left(\mathrm{RFA}_{\text {sub }}\right)$ variou de 0,06 para $0,56 \mathrm{~mol} \mathrm{~m}^{-2} \mathrm{dia}^{-1}$. Não houve diferença significativa nos valores de FCV entre os microssítios (Tabela 1), indicando que a abertura no dossel da floresta não variou entre as espécies. A temperatura no sub-bosque oscilou entre $22{ }^{\circ} \mathrm{C}$ à noite e $29{ }^{\circ} \mathrm{C}$ ao meio-dia, e a umidade média do ar foi superior a $90 \%$. Não houve diferença entre espécies nas taxas de crescimento em diâmetro e altura. A taxa de crescimento em diâmetro foi de $0,9 \mathrm{~mm}$ ano $^{-1}$, conquanto a taxa de crescimento em altura foi de 55,5 $\mathrm{mm}_{\text {ano }}{ }^{-1}$ (Tabela 1 ). Também, verificou-se aumento no incremento anual em diâmetro (IAD) das plantas com maior irradiância (Figura 1B).

\subsection{Nutrientes ( $\mathrm{N}$ e P), fotossíntese e área foliar específica}

Os teores de $\mathrm{N}$ variaram entre $15,7 \mathrm{mg} \mathrm{g}^{-1} \mathrm{em}$ T. unifoliolata e $22,4 \mathrm{mg} \mathrm{g}^{-1} \mathrm{em} \mathrm{S}$. guilleminiana. Por unidade de área, os conteúdos de $\mathrm{N}$ oscilaram entre $59,4 \mathrm{mmol} \mathrm{m}^{-2} \mathrm{em} \mathrm{T}$. unifoliolata e 123,5 $\mathrm{mmol} \mathrm{m}^{-2} \mathrm{em}$ S. guilleminiana (Tabela 1). Entretanto, os teores de P oscilaram entre $0,42 \mathrm{mg} \mathrm{g}^{-1}$ em C. duckeana $\mathrm{e}$ $0,58 \mathrm{mg} \mathrm{g}^{-1}$ em Z. juruana, enquanto o conteúdo de $\mathrm{P}$ por unidade de área variou entre $0,80 \mathrm{mmol} \mathrm{m}^{-2} \mathrm{em}$ E. amplum e 1,34 $\mathrm{mmol} \mathrm{m}^{-2} \mathrm{em} \mathrm{S}$. guilleminiana (Tabela 1). A eficiência no uso do $\mathrm{N}$ variou de 74,3 ( $\mu \mathrm{mol}\left(\mathrm{CO}_{2}\right) \mathrm{mol}^{-1}(\mathrm{~N}) \mathrm{s}^{-1}$ ) em L. oblongifolia a 135,3 $\left(\mu \mathrm{mol}\left(\mathrm{CO}_{2}\right) \mathrm{mol}^{-1}(\mathrm{~N}) \mathrm{s}^{-1}\right)$ em E. amplum. Já a eficiência

Revista Árvore, Viçosa-MG, v.37, n.4, p.707-716, 2013 
Tabela 1 - Incremento anual em diâmetro (IAD), incremento anual em altura (IAA), conteúdo de nitrogênio (N), conteúdo de fósforo (P), eficiência no uso do $\mathrm{N}$ e P, fração de céu visível (FCV) e valores SPAD, em 10 espécies florestais da Amazônia Central.

Table 1 - Annual increment in diameter (IAD, annual increment in height (IAA), nitrogen $(N)$ and phosphorus $(P)$ content and $N$ and $P$ use efficiency, fraction of sky visible (FCV) and SPAD values in 10 tree species of Central Amazonia.

\begin{tabular}{|c|c|c|c|c|c|c|c|c|c|c|}
\hline $\begin{array}{l}\text { Variáveis } \\
\text { espécies }\end{array}$ & $\begin{array}{c}\text { IAD } \\
\left(\mathrm{mm} \mathrm{ano}^{-1}\right)\end{array}$ & $\begin{array}{c}\text { IAA } \\
\left(\mathrm{mm} \mathrm{ano}^{-1}\right)\end{array}$ & $\begin{array}{c}\mathrm{N} \\
\left(\mathrm{mg} \mathrm{g}^{-1}\right)\end{array}$ & $\begin{array}{c}\mathrm{N} \\
\left(\mathrm{mmol} \mathrm{m}^{-2}\right)\end{array}$ & $\begin{array}{c}\mathrm{P} \\
\left(\mathrm{mg} \mathrm{g}^{-1}\right)\end{array}$ & $\begin{array}{c}\mathrm{P} \\
\left.(\mathrm{mmol} \mathrm{m})^{-2}\right)\end{array}$ & $\begin{array}{c}A_{\mathrm{pot}} / \mathrm{N}\left(A_{\mathrm{pot}} / \mathrm{N},\right. \\
\mu \mathrm{mol}\left(\mathrm{CO}_{2}\right) \\
\left.\mathrm{mol}^{-1}(\mathrm{~N}) \mathrm{s}^{-1}\right)\end{array}$ & $\begin{array}{c}A_{\mathrm{pot}} / \mathrm{P}\left(A_{\mathrm{pot}} / \mathrm{P}\right. \\
\mu \mathrm{mol}\left(\mathrm{CO}_{2}\right) \\
\left.\mathrm{mol}^{-1}(\mathrm{P}) \mathrm{s}^{-1}\right)\end{array}$ & FCV & $\begin{array}{c}\text { Valores } \\
\text { SPAD }\end{array}$ \\
\hline C. duckeana & $0,9 \mathrm{a}$ & $104,7 \mathrm{a}$ & $20,32 \mathrm{ab}$ & $89,43 \mathrm{abc}$ & $0,42 \mathrm{a}$ & $0,84 \mathrm{ab}$ & $81,3 \mathrm{ab}$ & 8880 a & $0,012 \mathrm{a}$ & $47,40 \mathrm{~b}$ \\
\hline E. guianensis & $0,8 \mathrm{a}$ & $44,7 \mathrm{a}$ & $19,18 \mathrm{ab}$ & $108,37 \mathrm{ab}$ & $0,44 \mathrm{a}$ & $1,09 \mathrm{ab}$ & $81,4 \mathrm{ab}$ & $7900 \mathrm{a}$ & $0,019 \mathrm{a}$ & $52,53 \mathrm{ab}$ \\
\hline E. amplum & $1,0 \mathrm{a}$ & $62,3 \mathrm{a}$ & $19,71 \mathrm{ab}$ & $68,94 \mathrm{bc}$ & $0,50 \mathrm{a}$ & $0,80 \mathrm{~b}$ & $135,3 \mathrm{a}$ & $12790 \mathrm{a}$ & $0,019 \mathrm{a}$ & $47,90 \mathrm{~b}$ \\
\hline L. oblongifolia & $0,8 \mathrm{a}$ & $67,0 \mathrm{a}$ & $17,19 \mathrm{ab}$ & 88,96 abc & $0,49 \mathrm{a}$ & $1,12 \mathrm{ab}$ & $74,3 \mathrm{~b}$ & $6020 \mathrm{a}$ & $0,011 \mathrm{a}$ & $43,63 \mathrm{~b}$ \\
\hline P. guianensis & $1,0 \mathrm{a}$ & $36,5 \mathrm{a}$ & $17,32 \mathrm{ab}$ & $78,27 \mathrm{abc}$ & $0,49 \mathrm{a}$ & $1,00 \mathrm{ab}$ & $112,6 \mathrm{ab}$ & $8760 \mathrm{a}$ & $0,012 \mathrm{a}$ & $50,96 \mathrm{~b}$ \\
\hline R. racemosa & $0,9 \mathrm{a}$ & $60,0 \mathrm{a}$ & $18,64 \mathrm{ab}$ & $62,48 \mathrm{bc}$ & $0,54 \mathrm{a}$ & $0,82 \mathrm{ab}$ & $121,3 \mathrm{ab}$ & 9480 a & $0,014 \mathrm{a}$ & $44,20 \mathrm{~b}$ \\
\hline S. guilleminiana & $0,9 \mathrm{a}$ & $78,8 \mathrm{a}$ & $22,38 \mathrm{a}$ & $123,48 \mathrm{a}$ & $0,54 \mathrm{a}$ & $1,34 \mathrm{a}$ & $74,9 \mathrm{~b}$ & $6840 \mathrm{a}$ & $0,015 \mathrm{a}$ & $64,26 \mathrm{a}$ \\
\hline T. unifoliolata & $1,3 \mathrm{a}$ & $69,4 \mathrm{a}$ & $15,74 \mathrm{~b}$ & $59,35 \mathrm{c}$ & $0,53 \mathrm{a}$ & $0,90 \mathrm{ab}$ & $123,9 \mathrm{ab}$ & $8230 \mathrm{a}$ & $0,014 \mathrm{a}$ & $54,23 \mathrm{ab}$ \\
\hline V. calophylla & $1,0 \mathrm{a}$ & $56,5 \mathrm{a}$ & $19,48 \mathrm{ab}$ & 88,89 abc & $0,48 \mathrm{a}$ & $0,98 \mathrm{ab}$ & $94,9 \mathrm{ab}$ & $8530 \mathrm{a}$ & $0,013 \mathrm{a}$ & $52,46 \mathrm{ab}$ \\
\hline Z. juruana & $0,7 \mathrm{a}$ & $48,2 \mathrm{a}$ & $19,22 \mathrm{ab}$ & 93,74 abc & $0,58 \mathrm{a}$ & $1,26 \mathrm{ab}$ & $89,5 \mathrm{ab}$ & $6770 \mathrm{a}$ & $0,012 \mathrm{a}$ & $52,35 \mathrm{ab}$ \\
\hline Média & 0,9 & 55,5 & 18,92 & 86,19 & 0,50 & 1,02 & 99,3 & 8420 & 0,014 & 50,99 \\
\hline CV (\%) & 56,2 & 44,1 & 9,81 & 19,39 & 16,6 & 17,85 & 18,86 & 30,83 & 39,17 & 8,27 \\
\hline
\end{tabular}

Médias seguidas de mesmas letras nas colunas não diferem de acordo com o teste de Tukey a 5\% de probabilidade.

Means followed by same letters in columns do not differ according to Tukey test at probability of $5 \%$.

no uso do P oscilou de $6.020\left(\mu \mathrm{mol}\left(\mathrm{CO}_{2}\right) \mathrm{mol}^{-1}(\mathrm{P}) \mathrm{s}^{-1}\right)$ em L. oblongifolia a $12.790\left(\mu \mathrm{mol}\left(\mathrm{CO}_{2}\right) \mathrm{mol}^{-1}(\mathrm{P}) \mathrm{s}^{-1}\right)$ em E. amplum (Tabela 1).

$A_{\text {pot-área }}$ foi positivamente correlacionada com o conteúdo de $\mathrm{N}$ e $\mathrm{P}(\mathrm{P} \leq 0,05)$ (Figura $2 \mathrm{AB})$. Entretanto, $A_{\text {pot-mas }}$ não apresentou relação significativa $(\mathrm{P}>0,05)$ com o conteúdo de $\mathrm{N}$ e $\mathrm{P}$ (Figura $2 \mathrm{CD}$ ). $A_{\text {pot-área }}$ não mostrou relação significativa com a eficiência no uso do $\mathrm{N}(\mathrm{P}>0,05$, Figura 2E), no entanto a maior eficiência no uso do $\mathrm{P}$ resultou em valores maiores de $A_{\text {pot-área }}$ ( $\mathrm{P} \leq 0,01$, Figura 2F). Observou-se que pequenas variações na irradiância do sub-bosque afetam significativamente o conteúdo de $\mathrm{N}$ e $\mathrm{P}(\mathrm{P} \leq 0,05$, Figura $3 \mathrm{AB})$. Entretanto, não houve efeito significativo entre irradiância do sub-bosque e os teores de $\mathrm{N}$ e $\mathrm{P}$ por unidade de massa $(P>0,05$, Figura $3 C D)$ e a eficiência no uso do N e P (P > 0,05, Figura 3E F).

Por unidade de área, os conteúdos de $\mathrm{N}$ e $\mathrm{P}$ foram positivamente relacionados (Figura $4 \mathrm{~A}$ ), mas não são expressos por unidade de massa ( $\mathrm{P}>0,05$, Figura 4B). A razão N/P foi positivamente relacionada com os teores de N (Figura 4CD), mas a relação entre N/P e os teores de $\mathrm{P}$ foi negativa (Figura 4EF). A eficiência no uso do $\mathrm{N}$ diminuiu com o aumento da razão N/P (Figura 4G), entretanto não houve relação entre a eficiência no uso do $\mathrm{P}$ e a razão N/P $(\mathrm{P}>0,05$, Figura $4 \mathrm{H})$. O conteúdo de $\mathrm{N}$ e P declinou significativamente com o aumento daAFE (Figura 5AB). Já a eficiência no uso do $\mathrm{N}$ aumentou linearmente com a AFE (Figura 5C). AFE e os conteúdos de $\mathrm{N}$ e $\mathrm{P}$ foram consistentemente relacionados com os valores de SPAD ( $\mathrm{P} \leq 0,01$, Figura 5D-F).

\section{DISCUSSÃO}

\subsection{Condições ambientais e crescimento das arvoretas}

Os valores de irradiância no sub-bosque observados neste estudo são similares aos descritos em outros trabalhos (MOLION, 1987; NASCIMENTO; MARENCO, 2013), que relataram que a luz no sub-bosque da floresta é inferior a $2 \%$ de irradiância registrada acima do dossel da floresta. Nas florestas tropicais úmidas, as baixas taxas de crescimento (diâmetro e altura) nas espécies de sub-bosque estão relacionadas à baixa luminosidade a que as árvores são expostas na fase juvenil. Em razões disso, pequenas variações nas aberturas e microaberturas do dossel infuenciam o incremento em diâmetro das arvoretas. A relação entre IAD e a irradiância observada neste estudo concorda com os resultados de Clark et al. (1999). As taxas de incremento anual em diâmetro (IAD) observadas neste estudo $\left(0,9 \mathrm{~mm} \mathrm{ano}^{-1}\right)$ são similares às médias de crescimento observadas em árvores adultas em florestas tropicais em torno de $1 \mathrm{~mm}^{-1}$ ano $^{-1}$ (CLARK; CLARK, 2001; VIEIRA et al., 2005). 

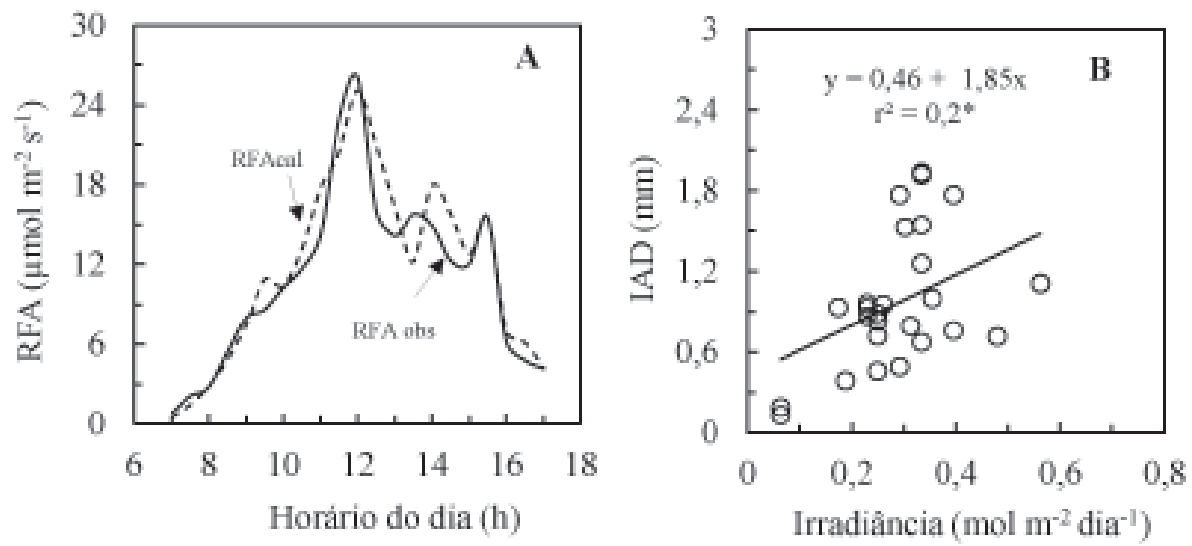

Figura 1 - Radiação fotossinteticamente ativa (RFA) no sub-bosque em torno de uma planta de L. oblongifolia selecionada ao acaso (A). Linha contínua: RFA observado $\left(\right.$ RFA $_{\text {obs }}$ ) e linha tracejada: RFA calculado (RFA ${ }_{\text {cal }}$ ). Relação entre o incremento anual em diâmetro (IAD) e a irradiância do sub-bosque (B), em 10 espécies florestais da Amazônia Central. Cada símbolo representa uma planta. *: significativo $(\mathrm{P}<0,05)$.

Figure 1 - Photosynthetically active radiation (RFA) in the understory around a plant of L. oblongifolia randomly selected (A). Continuous line: RFA observed $\left(R F A_{\text {obs }}\right)$ and dashed line: $R F A$ calculated $\left(R F A_{\text {cal }}\right)$. Relationship between the annual increment in diameter (IAD) and understory irradiance (B) in 10 forest tree species in Central Amazonia. Each symbol represents one plant. *: significant $(P<0.05)$.
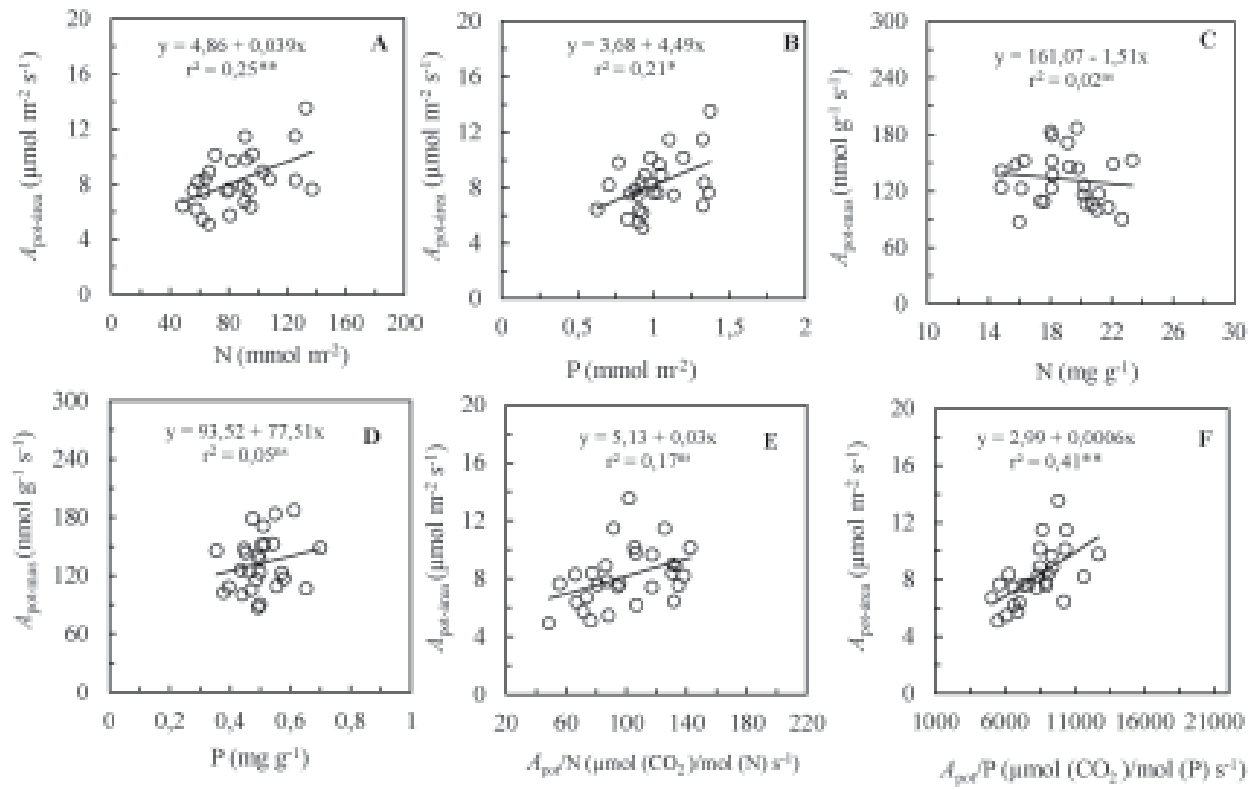

Figura 2 - Relação entre a capacidade fotossintética $(A$

A, B; $A$

, C, D) e conteúdo de nitrogênio $(\mathrm{N})$ e fósforo (P), eficiência no uso do nitrogênio $\left(A_{\text {pot }} / \mathrm{N} ; \mathbf{E}\right)$, eficiência no uso do fósforo $\left(A_{\text {pot }} / \mathrm{P} ; \mathbf{F}\right)$ em 10 espécies florestais da Amazônia Central. $A$ foi medido em $\left[\mathrm{CO}_{2}\right]$ de $2000 \mu \mathrm{mol} \mathrm{mol}^{-1}$, irradiância de $1.000 \mu \mathrm{mol} \mathrm{m}^{-2} \mathrm{~s}^{-1} \mathrm{e}$ temperatura foliar de $28 \pm 2{ }^{\circ} \mathrm{C}$. Cada símbolo representa uma planta. *: significativo $(\mathrm{P}<0,05)$; **: altamente significativo $(\mathrm{P}<0,01)$; e ns: não significativo $(\mathrm{P}>0,05)$.

Figure 2-Relationship between the photosynthetic capacity $\left(A_{\text {potarea, }} \boldsymbol{A}, \boldsymbol{B} ; A_{\text {pot-mas, }} \boldsymbol{C}, \boldsymbol{D}\right)$ and nitrogen $(N)$ and phosphorus $(P)$ content, nitrogen use efficiency $\left(A_{\text {pol }} / N, \boldsymbol{E}\right)$, phosphorus use efficiency $\left(A_{p o} / P, \boldsymbol{F}\right)$ in 10 tree species of Central Amazonia. $A_{\text {pot }}$ was measured at $\left[\mathrm{CO}_{2}\right]$ of $2000 \mathrm{\mu mol} \mathrm{mol}^{-1}$, irradiance of $1000 \mu \mathrm{mol} \mathrm{m} \mathrm{m}^{-2} \mathrm{~s}^{-1}$ and leaf temperature of $28 \pm 2{ }^{\circ} \mathrm{C}$. Each symbol represents one plant. *: significant $(P<0.05)$, **: highly significant $(P<0.01)$ and ns: not significant $(P>0.05)$. 

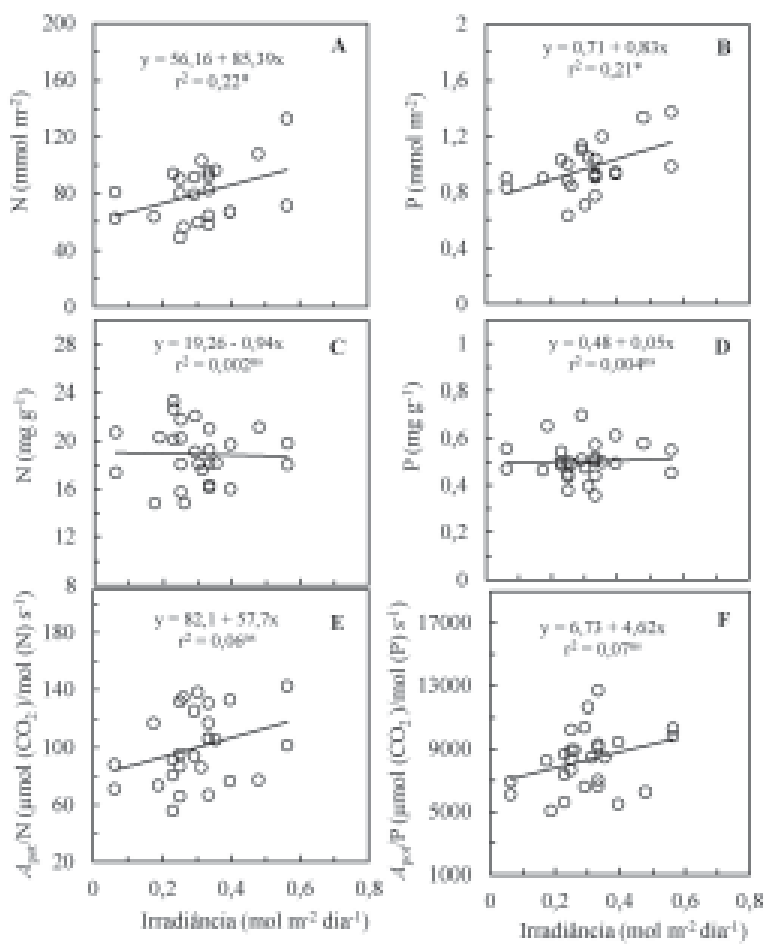

Figura 3 - Relação entre o conteúdo de nitrogênio e fósforo (unidade de área, A, B; unidade de massa, C, D), eficiência no uso do nitrogênio $\left(A_{\text {pot }} / \mathrm{N}\right.$; $\left.\mathbf{E}\right)$, eficiência no uso do fósforo $\left(A_{\text {pot }} / \mathrm{P} ; \mathbf{F}\right)$ e irradiância do subbosque em 10 espécies florestais da Amazônia Central. Cada símbolo representa uma planta. *: significativo ( $\mathrm{P}<0,05)$; e ns: não significativo $(\mathrm{P}>0,05)$.

Figure 3 - Relationship between nitrogen and phosphorus content (per unit area, $\boldsymbol{A}, \boldsymbol{B} ;$ per unit mass, $\boldsymbol{C}$, $\boldsymbol{D})$, nitrogen use efficiency $\left(A_{\text {pot }} / N, \boldsymbol{E}\right)$, phosphorus use efficiency $(A, P, \boldsymbol{F})$ and understory irradiance in 10 tree species of Central Amazonia. Each symbol represents one plant. *: significant $(P<0.05)$ and ns: not significant $(P>0.05)$.

Porém, os valores de IAD deste artigo são menores do que os observados em arvoretas cultivadas em baixa luminosidade e com a adição de nutrientes (AZEVEDO; MARENCO, 2012). A taxa média de crescimento em altura de $55,5 \mathrm{~mm} \mathrm{ano}^{-1}$ indica que as arvoretas utilizadas no estudo ( 1 a $3 \mathrm{~m}$ ) podem levar mais de 200 anos para atingir o dossel da floresta, assumindo uma taxa de crescimento constante. Evidentemente, esse tempo pode ser menor, haja vista que, à medida que as árvores crescem, elas passam a receber mais luz. No entanto, considerando o incremento em diâmetro as arvoretas deste estudo levariam aproximadamente 100 anos para atingir um diâmetro de $100 \mathrm{~mm}$, o que estaria de acordo com Clark e Clark (2001), que estimaram que as arvoretas do sub-bosque possam levar mais de 80 anos para atingir o dossel.

\subsection{Nutrientes ( $\mathrm{N}$ e $\mathrm{P}$ ), fotossíntese $\mathrm{e}$ área foliar específica}

Os conteúdos de $\mathrm{N}$ e $\mathrm{P}$ foram menores (Tabela 1) que os valores relatados em florestas tropicais por outros autores (RAAIMAKERS, 1995; REICH et al., 1999; OSNAS et al., 2013). Diversos estudos têm mostrado a importância do N e P na fotossíntese (REICH et al., 1999; FYLLAS et al., 2009), no entanto há pouca informação sobre os níveis críticos e ótimos de nutrientes para espécies florestais da Amazônia. Em Swietenia macrophylla e Brosimum alicastrum, conteúdos de nutrientes foliares abaixo de 10-14 $\mathrm{mg} \mathrm{g}^{-1}$ (para N) e $0,6 \mathrm{mg} \mathrm{g}^{-1}$ (para P) são considerados deficientes para o crescimento (DRECHSEL; ZECH, 1991). Assim, em comparação com o $\mathrm{N}$, os baixos conteúdos de $\mathrm{P}$ foliar e a alta relação N/P (KOERSELMAN; MEULEMAN, 1996) sugerem que o $P$ tem maior chance de ser elemento limitante nas espécies estudadas. Os resultados deste estudo são diferentes daqueles relatados por Reich et al. (1991), que observaram relação significativa entre fotossíntese e $\mathrm{N}$ apenas por unidade de massa foliar.

A fotossíntese está relacionada à variação do conteúdo de $\mathrm{N}$, pois aproximadamente $60 \%$ do nitrogênio total da folha encontra-se nos cloroplastos, principalmente formando parte de enzimas da fotossíntese, como a Rubisco (HIKOSAKA, 2004). Segundo Reich et al. (1998), a relação entre as taxas fotossintéticas e $\mathrm{N}$ expresso por unidade de área ocorre, principalmente, quando há pequena variação no conteúdo de $\mathrm{N}$ por unidade de massa, o que talvez possa explicar a falta de relação entre $A_{\text {pot-mas }}$ e os teores de N e P. Ainda, deve-se notar (Figura 2B) que a relação entre $A_{\text {pot-área }}$ e $\mathrm{P}$ foi um pouco menos estreita $\left(\mathrm{r}^{2}=0,21\right)$ do que a relação entre $A_{\text {pot-área }}$ e N ( $\mathrm{r}^{2}=0,25$; Figura 2A), indicando que, em comparação com o $\mathrm{P}$, maior fração de $\mathrm{N}$ na folha forma parte integral das estruturas fotossintéticas.

Os valores da eficiência no uso do $\mathrm{N}$ e P foram maiores do que os observados por outros autores (HIREMATH, 2000; CORDELL et al., 2001). Vitousek e Howarth (1991) relataram que, em geral, plantas que crescem em solos pobres em nutrientes podem apresentar maior eficiência de uso dos nutrientes do que plantas das mesmas espécies crescendo em solos mais ricos 

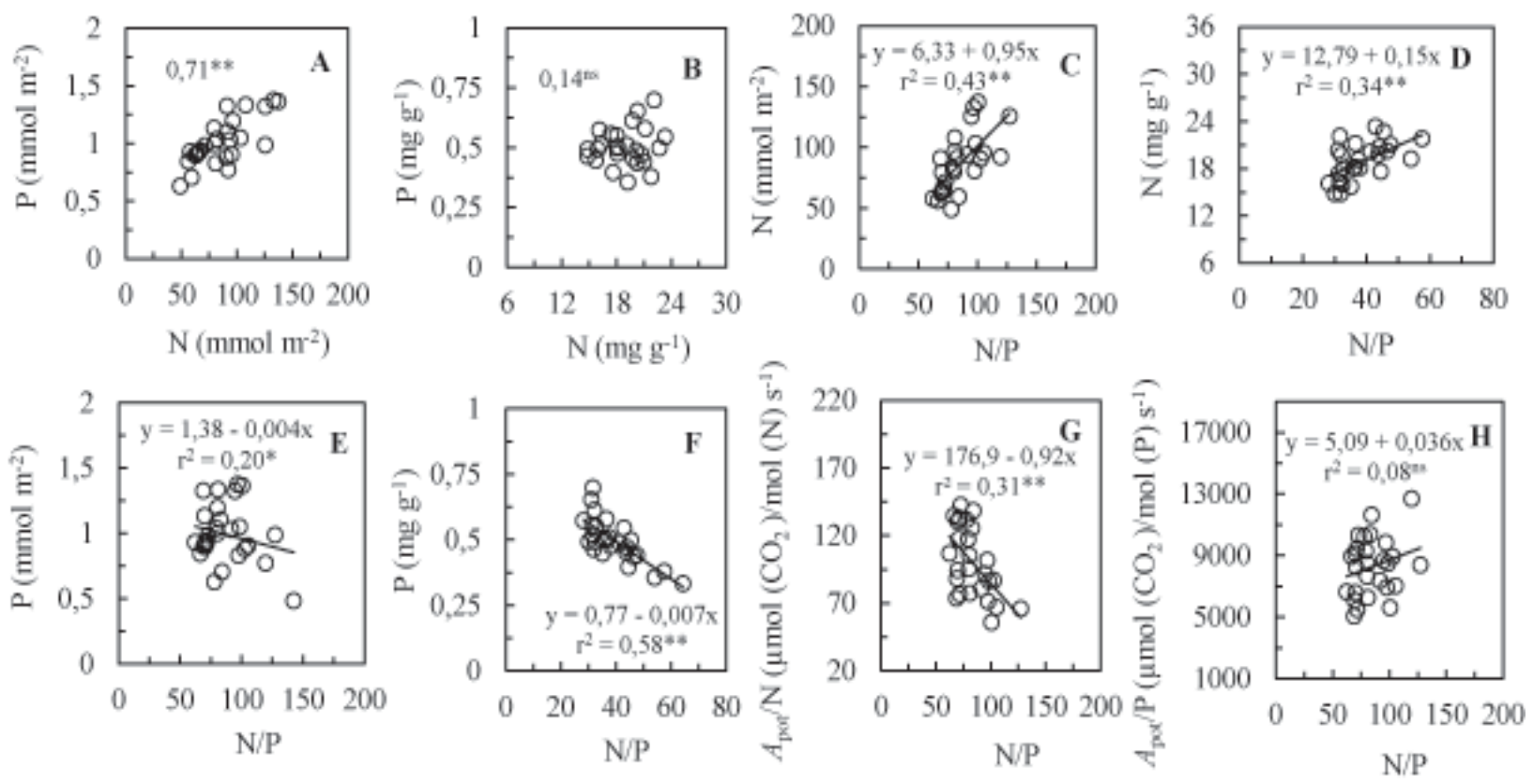

Figura 4 - Relação entre conteúdo de nitrogênio (N) e fósforo (P) $(\mathbf{A}, \mathbf{B}), \mathrm{N}$ e $\mathrm{P}$ e a razão N/P $(\mathbf{C}, \mathbf{D}, \mathbf{E}, \mathbf{F})$, eficiência no uso do nitrogênio $\left(A_{\text {pot }} / \mathrm{N}, \mathbf{G}\right)$, eficiência no uso do fósforo $\left(A_{\mathrm{pot}} / \mathrm{P}, \mathbf{H}\right)$ e razão N/P, em 10 espécies florestais da Amazônia Central. Cada símbolo representa uma planta. *: significativo $(\mathrm{P}<0,05)$; $* *$ : altamente significativo $(\mathrm{P}<0,01)$; e ns: não significativo $(\mathrm{P}>0,05)$.

Figure 4 - Relationship between nitrogen $(N)$ and phosphorus $(P)$ content $(\boldsymbol{A}, \boldsymbol{B}), N$ and $P$ and the $N / P$ ratio $(\boldsymbol{C}, \boldsymbol{D}, \boldsymbol{E}$, F), $N$ use efficiency $\left(A_{\text {pot }} / N, \boldsymbol{G}\right), P$ use efficiency $\left(A_{\text {pot }} / P, \boldsymbol{H}\right)$ and the $N / P$ ratio in 10 tree species of Central Amazonia. Each symbol represents one plant. *: significant $(P<0.05), * *$ : highly significant $(P<0.01)$ and ns: not significant $(P>0.05)$.

em nutrientes. A relação positiva entre $A_{\text {pot }} \mathrm{e} A_{\text {pot }} / \mathrm{P}$ e a falta de relação entre $A_{\mathrm{pot}} \mathrm{e} A_{\mathrm{pot}} / \mathrm{N}$ indica que, embora esses dois elementos influenciem significativamente a capacidade fotossintética da folha, os teores mínimos do N são comparativamente maiores do que os teores mínimos de P. Isso sugere que, desses dois elementos, o nutriente que mais provavelmente limita a capacidade fotossintética da folha é o fósforo.

A tendência da irradiância no sub-bosque para influenciar os conteúdos de N e P (Figura 3) é consistente com o efeito da luminosiade na área foliar específia (folhas mais espessas tem maior teor de nutrientes por unidade de área). Isso está de acordo com os resultados relatados por Lloyd et al. (2010), que observaram acréscimo das concentrações de $\mathrm{N}$ e $\mathrm{P}$ com o aumento da luminosidade do dossel.

A falta de correlação entre $A_{\text {pot }} / \mathrm{N}, A_{\text {pot }} / \mathrm{P}$ e a irradiância sugere que, na faixa de intensidade de luz observada no sub-bosque $\left(0,06\right.$ a $\left.0,56 \mathrm{~mol} \mathrm{~m}^{-2} \mathrm{dia}^{-1}\right)$, a fotossíntese é mais influenciada pelo conteúdo de nutrientes minerais do que pela irradiância. Isso está de acordo com Poorter e Evans (1998), que observaram maior eficiência no uso de $\mathrm{N}$ em alta irradiância.

O conteúdo de $\mathrm{N}$ por unidade de área aumentou linearmente com o conteúdo de $\mathrm{P}$ (Figura 4A). Resultados semelhantes também foram descritos por outros (GÜSEWELL; KOERSELMAN, 2002; GÜSEWELL, 2004). Isto é consistente com os aumentos dos teores de $\mathrm{N}$ e $\mathrm{P}$ com o aumento da espessura da folha (menor AFE).

Em contrapartida, o conteúdo de $\mathrm{N}$ e $\mathrm{P}$ expresso por unidade de massa não foi correlacionado (Figura 4B). Esses resultados estão de acordo com os de Niinemets e Kull (2003), que não encontraram correlação entre as concentrações de $\mathrm{N}$ e $\mathrm{P}$ em espécies que apresentaram pouca variabilidade nos teores desses elementos. O conteúdo de $\mathrm{N}$ aumentou consistentemente com a razão N/P. Entretanto, o 
conteúdo de $\mathrm{P}$ foliar e a razão N/P correlacionaram-se negativamente (Figura 4). Isso está de acordo com Güsewell (2004), que observou relação negativa entre N/P e a concentração de P. Reich e Schoettle (1988) observaram que a eficiência no uso do $\mathrm{N}$ aumentou linearmente com $\mathrm{N} / \mathrm{P}$, contrário ao relatado neste trabalho (Figura 4G). Isso indica que nas plantas
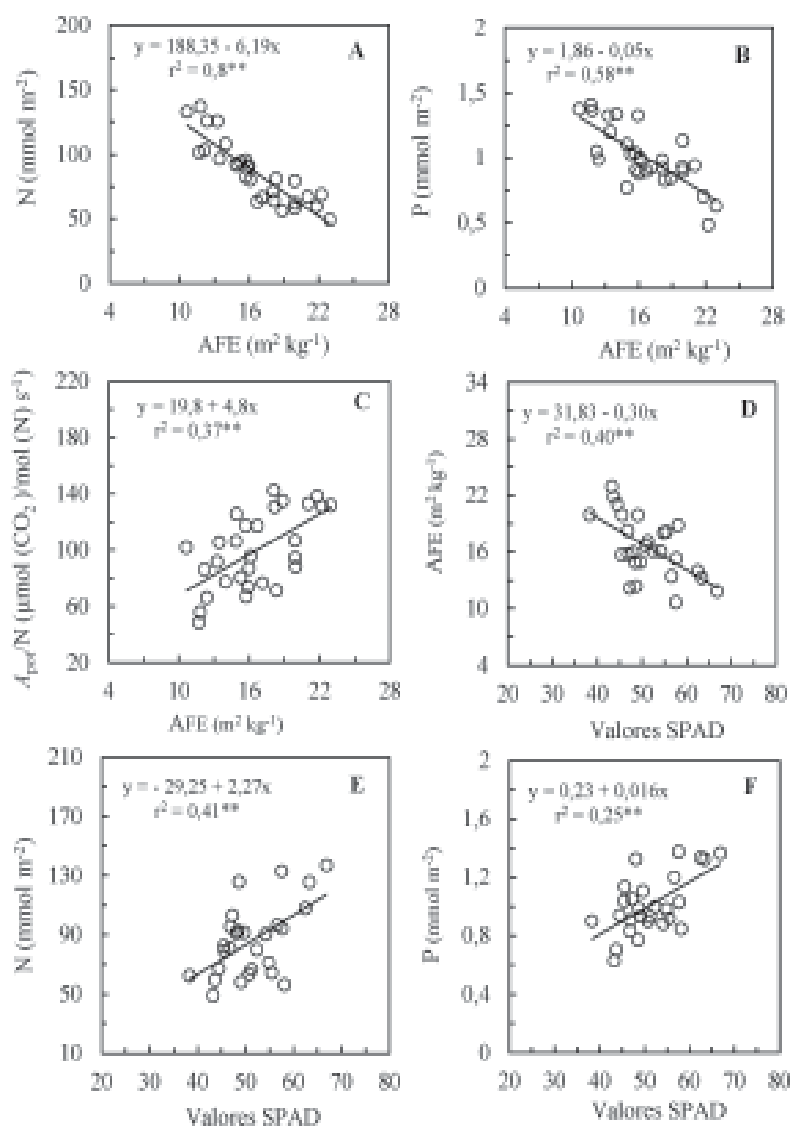

Figura 5 - Relação entre o conteúdo de nitrogênio (N) e fósforo $(\mathrm{P})(\mathbf{A}, \mathbf{B})$, eficiência no uso do nitrogênio $\left(A_{\text {pot }} / \mathrm{N} ; \mathbf{C}\right)$ e área foliar específica (AFE). Relação entre área foliar específica (AFE; D), conteúdo de nitrogênio $(\mathrm{N})$ e de fósforo $(\mathrm{P})(\mathbf{E}, \mathbf{F})$ e valores SPAD, em 10 espécies florestais da Amazônia Central. Cada símbolo representa uma planta. **: altamente significativo ( $\mathrm{P}<0,01)$.

Figure 5 - Relationship between nitrogen $(N)$ and phosphorus $(P)$ content $(\boldsymbol{A}, \boldsymbol{B})$, nitrogen use efficiency $(A / N$, C), and specific leaf area (SLA). Relationship between specific leaf area (AFE; D), nitrogen $(N)$ and phosphorus $(P)$ content $(\boldsymbol{E}, \boldsymbol{F})$ and $S P A D$ values in 10 tree species of Central Amazonia. Each symbol represents one plant. **: highly significant $(P<0.01)$. deste estudo a eficiência no uso do $\mathrm{N}$ dimimui substancialmente à medida que aumenta as concentrações de $\mathrm{P}$, o que novamente sugere que o fósforo é o elemento mais crítico para o crescimento das árvores. Essa hipótese é consistente com o fato de a relação entre $A_{\text {pot }} / \mathrm{P}$ e N/P não ser significativa, ou seja, o aumento na concentração de $\mathrm{N}$ não altera significativamente a eficiência no uso do P.

A diminuição na AFE está frequentemente associada com o aumento no conteúdo de nitrogênio e fósforo por unidade de área foliar (POORTER; EVANS, 1998; REICH et al., 1999; OSONE et al., 2008), devido, em parte, ao maior acúmulo de proteínas fotossintéticas. A eficiência no uso do $\mathrm{N}$ foi mais elevada nas espécies com maior área foliar específica, corroborando os resultados de Poorter e Evans (1998) e Reich et al. (1998). Esses autores indicaram que folhas mais finas (maior AFE) fazem melhor uso do $\mathrm{N}$ disponível, embora folhas mais finas tenham menor teor de clorofila por unidade de área e, consequentemente, menores valores SPAD (MARENCO et al., 2009) e menores teores de nitrogênio e fósforo por unidade de área. Chang e Robison (2003) também observaram maiores valores de SPAD em folhas com maior teor de N. Os resultados deste trabalho apontam a necessidade de se efetuarem estudos in loco envolvendo a adição de fósforo ao solo para quantificar o efeito do aumento na disponibilidade desse nutriente no crescimento e fisiologia das árvores.

\section{CONCLUSÃO}

Pequenas alterações na abertura do dossel afetam o incremento em diâmetro das arvoretas e os conteúdos de $\mathrm{N}$ e $\mathrm{P}$ foliar por unidade de área, mostrando alta sensibilidade das árvores na fase juvenil às variações no ambiente luminoso. Os baixos teores foliares de $\mathrm{P}\left(0,3\right.$ a $\left.0,6 \mathrm{mg} \mathrm{g}^{-1}\right)$ e a alta relação N/P (30 a 50), bem como a estreita relação entre as taxas de fotossíntese e a eficiência no uso do $\mathrm{P}$, indicam que este elemento é utilizado com alta eficiência nas plantas da Amazônia Central.

\section{AGRADECIMENTOS}

Ao Ministério da Ciência e Tecnologia MCT/INPA, à Fundação de Amparo à Pesquisa do Estado do Amazonas -FAPEAM (projeto UA 062.03164/2012), à CAPES e ao CNPq. 


\section{REFERÊNCIAS}

AZEVEDO, G. F. C.; MARENCO, R. A. Growth and physiological changes in saplings of Minquartia guianensis and Swietenia macrophylla during acclimation to full sunlight. Photosynthetica, v.50, n.1, p.86-94, 2012.

BARROSO, D. G. et al. Diagnóstico de deficiências de macronutrientes em mudas de teca. Revista Árvore, v.29, n.5, p.671-679, 2005.

CHANG, S. X.; ROBISON, D. J. Nondestructive and rapid estimation of hardwood foliar nitrogen status using the SPAD-502 chlorophyll meter. Forest Ecology and Management, v.181, n.3, p.331-338, 2003.

CLARK, D. A.; CLARK, D. B. Getting to the canopy: Tree height growth in a neotropical rainforest. Ecology, v.82, n.5, p.1460-1472, 2001.

CLARK, D. B.; PALMER, M.; CLARK, D. A. Edaphic factors and the landscape-scale distribution of tropical rain forest trees. Ecology, v.80, n.8, p.2662-2675, 1999.

CORDELL, S. et al. Regulation of leaf life-span and nutrient-use efficiency of Metrosideros polymorpha trees at two extremes of a long chronosequence in Hawaii. Oecologia, v.127, n.2, p.198-206, 2001.

COX, P. M. et al. Amazonian forest dieback under climate carbon cycle projections for the $21 \mathrm{st}$ century. Theoretical and Applied Climatology, v.78, n.1, p.137-156, 2004.

DENSLOW, J. S. et al. Growth responses of tropical shrubs to treefall gap environments. Ecology, v.71, n.1, p.165-179, 1990.

DRECHSEL, P.; ZECH, W. Foliar nutrient levels of broadleaved tropical trees: a tabular review. Plant and Soil, v.131, n.1, p.29-46, 1991.

DIAS, D. P. Fotossíntese e crescimento em diâmetro de árvores em função da temperatura e da precipitação numa floresta primária de terrafirme na Amazônia Central. 2009. 122f. Tese (Doutorado em Ciências de Florestas Tropicais) Instituto Nacional de Pesquisas da Amazônia, Manaus, 2009.
FYLLAS, N. M. et al. Basin-wide variations in foliar properties of Amazonian forest: phylogeny, soils and climate. Biogeosciences, v.6, n.4, p.2677-2708, 2009.

GÜSEWELL, S. N:P ratios in terrestrial plants: variation and functional significance. New Phytologist, v.164, n.2, p.243-266, 2004.

GÜSEWELL, S.; KOERSELMAN, W. Variation in nitrogen and phosphorus concentrations of wetland plants. Perspectives in Ecology, Evolution and Systematics, v.5, n.1, p.37$61,2002$.

HIKOSAKA, K. Interspecific difference in the photosynthesis-nitrogen relationship: patterns, physiological causes, and ecological importance. Journal of Plant Research, v. 117, n.6, p.481-494, 2004.

HIREMATH, A. J. Photosynthetic nutrient-use efficiency in three fast-growing tropical trees with differing leaf longevities. Tree Physiology, v.20, n.14, p.937-944, 2000.

KITAJIMA, K.; MULKEY, S. S.; WRIGHT, S. J. Decline of photosynthetic capacity with leaf age in relation to leaf longevities for five tropical canopy tree species. American Journal of Botany, v.84, n.5, p.702-708, 1997.

KOERSELMAN, W.; MEULEMAN, A. F. M. The vegetation $N$ :P ratio: a new tool to detect the nature of nutrient limitation. Journal of Applied Ecology, v.33, n.6, p.1441-1450, 1996.

KÖPPEN, W. Das geographische system der klimate. In: KOPPEN, W.; GEIGER, R. (Ed.) Handbuch der Klimatologie. Berlin: Gebrüder Bornträger, 1936. p.C1-C44.

LLOYD, J. et al. Optimisation of photosynthetic carbon gain and within-canopy gradients of associated foliar traits for Amazon forest trees, Biogeosciences, v.7, n.6, p.1833-1859, 2010.

LAWLOR, D. W.; CORNIC, G. Photosynthetic carbon assimilation and associated metabolism in relation to water deficits in higher plants. Plant Cell and Environment, v.25, n.2, p.275-294, 2002.

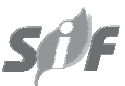

Revista Árvore, Viçosa-MG, v.37, n.4, p.707-716, 2013 
MARENCO, R. A.; ANTEZANA-VERA, S. A.; NASCIMENTO, H. C. S. Relationship between specific leaf area, leaf thickness, leaf water content and SPAD-502 readings in six Amazonian tree species. Photosynthetica, v.47, n.2, p.184190, 2009.

MENDES, K. R.; MARENCO, R. A. Leaf traits and gas exchange in saplings of native tree species in the Central Amazon. Scientia Agricola, v.67, n.6, p.624-632, 2010.

MOLION, L. C. B. Micrometeorology of an Amazonian rain forest. In: DICKINSON, R. E. (Ed.) The geophysiology of Amazonia: vegetation and climate interactions. New York: John Wiley, 1987. p.255-272.

NASCIMENTO, H. C. S.; MARENCO, R. A. Mesophyll conductance variations in response to diurnal environmental factors in Myrcia paivae and Minquartia guianensis in Central Amazonia. Photosynthetica, v. 51, n.3, p.457-464, 2013

NEVES, O. S. C. et al. Crescimento, produção de matéria seca e acúmulo de N, P, K, CA, MG e S na parte aérea de mudas de andiroba (Carapa guianensis Aubl.) cultivadas em solo de várzea, em função de diferentes doses de fósforo. Revista Árvore, v.28, n.3, p.343-349, 2004.

NIINEMETS, U.; KULL, K. Leaf structure vs. nutrient relationships vary with soil conditions in temperate shrubs and trees. Acta Oecologica, v.24, p.209-219, 2003.

OSNAS, J. L. D. et al. Global leaf trait relationships: mass, area, and the leaf economics spectrum. Science, v.340, n.6133, p.741-744, 2013.

OSONE, Y.; ISHIDA, A.; TATENO, M. Correlation between relative growth rate and specific leaf area requires associations of specific leaf area with nitrogen absorption rate of roots. New Phytologist, v.179, n.2, p.417-427, 2008.
POORTER, H.; EVANS, J. R. Photosynthetic nitrogen-use efficiency of species that differ inherently in specific leaf area. Oecologia, v. 116, n. 1, p. 26-37, 1998.

RAAIMAKERS, D. et al. Photosynthetic rates in relation to leaf phosphorus content in pioneer versus climax tropical rainforest trees.

Oecologia, v.102, n.1, p.120-125, 1995.

REICH, P. B.; ELLSWORTH, D. S.; WALTERS, M. B. Leaf structure (specific leaf area) modulates photosynthesis-nitrogen relations: evidence from within and across species and functional groups. Functional Ecology, v.12, n.6, p.948-958, 1998.

REICH, P. B. et al. Generality of leaf trait relationships: A test across six biomes. Ecology, v.80, n.6, p.1955-1969, 1999.

REICH, P. B.; SCHOETTLE, A. W. Role of phosphorus and nitrogen in photosynthetic and whole plant carbon gain and nutrient use efficiency in eastern white pine. Oecologia, v.77, n.1, p.25-33, 1988.

REICH, P. B. et al. Leaf lifespan as a determinant of leaf structure and function among 23

Amazonian tree species. Oecologia, v.86, n.1, p.16-24, 1991.

SILVA, F. C. Manual de análises químicas de solos, plantas e fertilizantes. 2.ed. Brasília: Embrapa Informação Tecnológica, 2009. 627p.

VIEIRA, S. et al. Slow growth rates of Amazonian trees: consequences for carbon cycling.

Proceedings of the National Academy of Sciences of the United States of America, v.102, n.51, p.18502-18507, 2005.

VITOUSEK, P. M.; HOWARTH, R. W. Nitrogen limitation on land and in the sea: How can it occur? Biogeochemistry, v.13, n.1, p.87-115, 1991. 